\title{
Identification of the Inrush Current of The Internal Faults of Power Transformers Based on the Differential Relay Function
}

\author{
Majid Salimi \\ Department of Electrical Engineering, Islamic Azad University, Tonekabon, Iran.
}

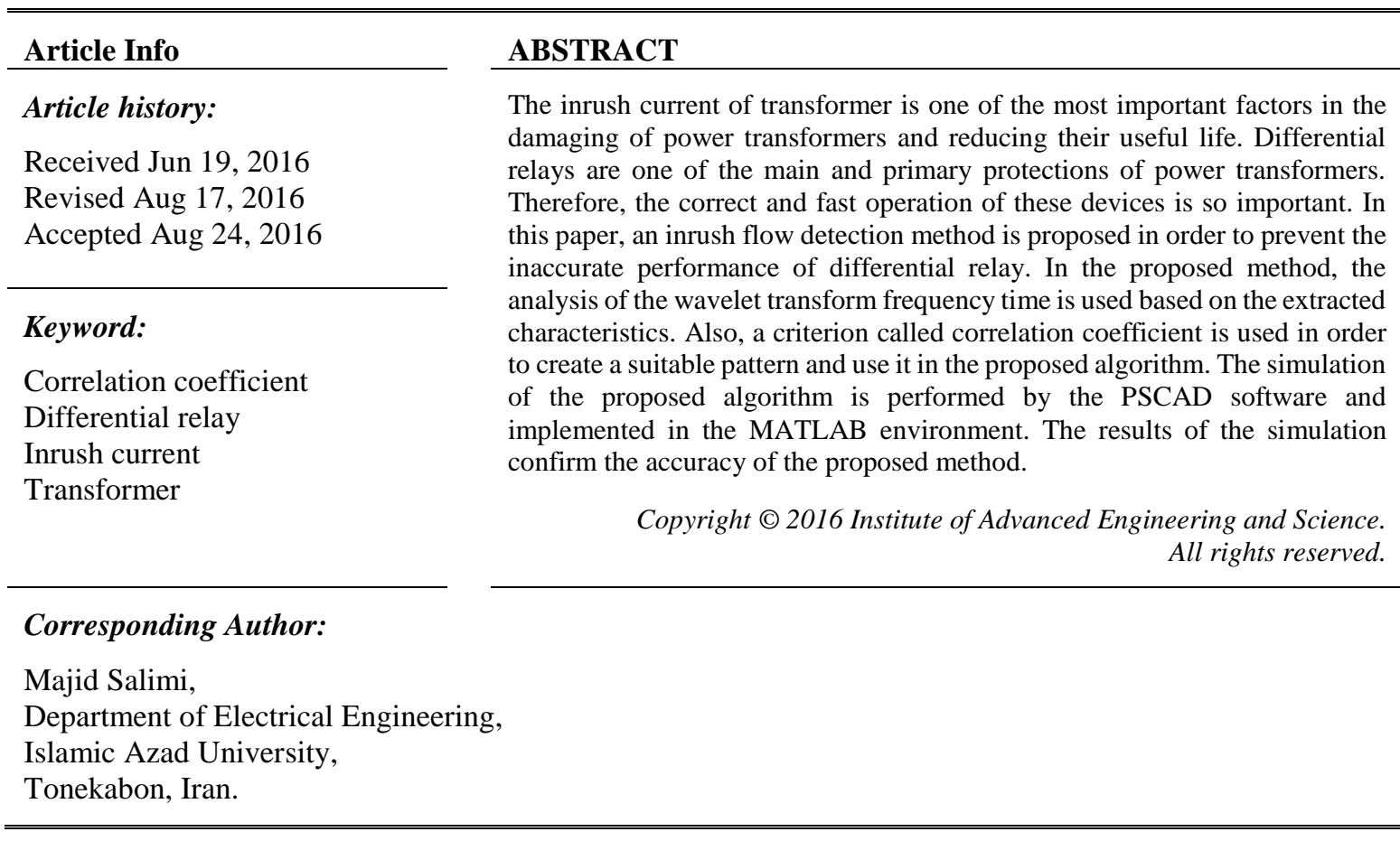

\section{INTRODUCTION}

The correct and stable performance of the power transformer plays a significant role in the stability of the power system and its attached devices. Any fault in the power transformer affects the power system connected to it. Therefore, the correct diagnosis of the fault and the rapid interruption of the damaged power transformer from the rest of the power system is important to reduce the damage caused by the severe flow of the fault and to prevent the power system instability. Differential protection is one of the most important and primary protection of the power transformers [1]. The differential protection as the main protection of the Power transformers has been considered for many years. However, this protection suffers from a basic weakness which is the detection of an error current from an inrush current [2]. Currently, the method mainly used for this purpose is the diagnostic method based on the second harmonic. However, with the use of highquality materials and the reduction of the transform core losses in the power transmissions, the second harmonic was reduced in the inrush current and its value was equal to or even less than the second harmonic, which leads to the unacceptable results of the algorithm in detecting the inrush current from the error current.

Some methods, including the method of limiting magnetic flux and its equivalent circuit, are used as a basic method for detecting the inrush current from the error current. In some papers, internal errors are directly detected by distortion of the waveform of the differential current, such as waveform correlation analysis, which detects the inrush current by using the waveform coefficient between the first half cycle and the second half cycle [3]. But the operation time of these methods is longer than a cycle and leads to a relatively long operation. Some other articles focus on the benefits of using the transient current flux transform and its analysis; however, these methods have the complexity of computational operations, and CT saturation is not considered [4]. Moreover, in combination with the artificial neural network method and fuzzy mathematical theory, in some researches this method based on the wavelet analysis is used for detecting the inrush current. 
However, the network design and the fuzzy rules require a large number of comprehensive training examples, which all of them are derived from a real power system, so that they cannot be easily used in practical applications [5]. On the other hand, mathematical morphology (MM) has been introduced as a powerful tool of flow waveform processing in the detection of the inrush current from the error current, and the article presents a simple and effective design using MM, but it may be unreliable about CT saturation [6]. However, this method fails when the transient internal fault and the inrush current occurs simultaneously. In this paper, we will investigate the detection of the inrush current from internal fault of power transformers based on the performance of differential relays.

\section{Research Hypotheses:}

- The converter is connected to the secondary coil without any charge

- The inrush current flows only in the primary coil

- $\quad$ No losses are considered in the power network.

\section{LITERATURE REVIEW}

In a research, a new and general rule has been introduced that distinguishes an inrush current from the error current using morphology and based on an algorithm in the time domain. At the same time, the transformer model has been used with sufficient accuracy to analyze the inrush current and the short-circuit current with different magnetization curves. The proposed method is based on the ratio of three-phase current and flows of the power transformers during the power supplying and the error conditions. The results of simulations carried out by MATLAB / EMTP software confirm the ability of the proposed method to distinction and detection in the time of half a cycle of power frequency [7]. An algorithm is proposed to identify and distinguish the inrush and error currents of power transformers. The first three-phase flows of the transformer and the integrals of the three-phase voltage of transformer are used in this proposed method. According to the proposed method, in the case of tantalizing the unloaded transformer, the correspond three-phase flows and the three-phase fluxes (the secondary three-phase voltage integrators) with the high accuracy (the synchronization difference is about 10 samples of the 1000-sample data window) have synchronization.

In this regard, the time difference between the maximum points of the three-phase current and flux and the time difference between the minimum points of the three-phase current and flux are considered as six distinct characteristics. The simulation results with the MATLAB/ EMTP software in the presence of different conditions of the inrush current (different types of cores with different curves and the alternating times of power supplying in a cycle) and the error current (different errors with different type, resistance, and start time) indicate the high precision and error-free of the proposed algorithm in the distinction between these two overcurrent. Another advantage of the proposed method is the detection speed of the algorithm which is the time less than half a cycle of power frequency $(10 \mathrm{~ms})$, [8]. In one the previous studies, two methods based on the artificial intelligence have been used to detect the inrush currents from the error currents. Initially, two different forms of group-based algorithms, GSA and PSO, were used to train the neural network.

The GSA acts on the basis of the gravity law and unlike the other algorithms based on the group of particles, and also is independent of the initial position of particles, and the particles are identities, and the PSO is based on the group movement of birds. Then, the neural network is going to be trained to detect the inrush current from the error current. In order to demonstrate that this training method is useful, and it leads to more accurate results, the results obtained from two proposed algorithms and the back-propagation (BP) methods, which is one of the most common methods for training of the neural networks, were compared [9]. When a power transformer is connected to a power supply, at the moment of the connection a very large current is created in the front side of transverse, which is much larger than the transformer's rated current. This phenomenon is called a Magnetizing inrush current, which results in inappropriate performance of protective systems. In fact, the similarity between the inrush current's characteristics and the internal error conditions causes this error. Therefore, for safe operation of the transformer, it is necessary to recognize the inrush current from the error current [10]. In this project, the neural network has been used to detect the inrush current from the internal error current.

A new collective algorithm, called the GSA, is presented for training in the neural network. In order to show the quality and ability of the proposed algorithm, the results obtained from the GSA algorithm are compared with the results obtained from PSO and BP algorithm which is one of the most common methods for training the neural network. The results show that the proposed algorithm (GSA) reduces the testing and the training time [11]. In the further study, a new method for detecting the inrush current from the internal error current of the power transformer is presented. In the proposed method of this paper, the wavelet transformation is used to analyze the current signal to the approximation coefficients and the detailed coefficients. Then, the determination of the current is based on a criterion determined by these coefficients. In this method, a statistical 
criterion called the correlation coefficient is used to investigate and recognition of the relationship between the energy of wavelet transformation coefficients at different levels of decomposition. Then, the diagnostic criterion is made using these correlation coefficients. The validity of the proposed algorithm is investigated using the signals of the simulated inrush current and the error flow. The simulation results demonstrate the ability of the proposed algorithm to detect the inrush current from the internal error current of the transformer [12-13].

\section{Research objectives}

a. Reviewing and comparing a variety of methods for detecting the inrush current.

b. Investigation and modeling of power network considering the effect of internal error and the inrush current of the power transformer.

c. Implementing the algorithm used in differential relay

d. Providing an effective indicator in the separation of the inrush current from the internal error current of the transformer.

e. Validation of the results obtained from the presented method with the old method's results.

\section{METHODOLOGY}

Since the method of this research can be used practically, is an applied research. Variable protection is the main protection scheme for large converters, especially those with a capacity more than 10 MVA. These converters play a major role in the operation of electrical power systems. It's obvious high importance and cost is required to the development and completion of a reliable protective system with the ability to detect internal errors and disconnect the equipment as quickly as possible. In the variable-protection converters, the main problem is in identifying the inrush currents from the internal short-circuit currents. Typically, variable relays perform this function using a harmonic support technique based on the assumption that the inrush currents have a high concentration on the second-order harmonic components. However, this technique is not always effective. In this research, a new method based on a variable current gradient for the separation between the inrush current and the internal errors current in the three-phase power converters will be tested. The proposed technique will be evaluated in a trial system of the three-phase power converter $25 \mathrm{MVA}, 13.8$ / $138 \mathrm{KV}$ and using MATLAB software. MATLAB software is used in this paper to simulate the inrush and internal error current. In all simulations, the $\Lambda \mathrm{SABC}$ threshold is set to 3 degrees.

\section{NUMERICAL EXAMPLES AND THE GENERAL SCHEMA OF THE PROBLEM AND THE STUDYING SYSTEM}

In order to test the performance of the proposed algorithm and its efficiency for development in the power systems, considering the new presented constraints, it is necessary first of all that the proper test issues be designed according to the real-world distribution network, the main parameters of the problem in different conditions are tested and the best possible solution for the problem is considered. Then, to validate it, we compared the result with an innovative or meta-innovative algorithm. Above mentioned issues will be explained in the following. To test the validity of the proposed method, a typical power system is used. This power system consists of a three-phase source KV 220 which is connected to a three-phase transformer and two coil through a transmission line KM 20. The neutral points on the star's connection on both sides of the transformer were landed. Also, the secondary transformer is connected to a three-phase charge of 44-ohm, and 0.068 henri. The following figure shows the single-line diagram of the sample power system as shown in Figure 1.

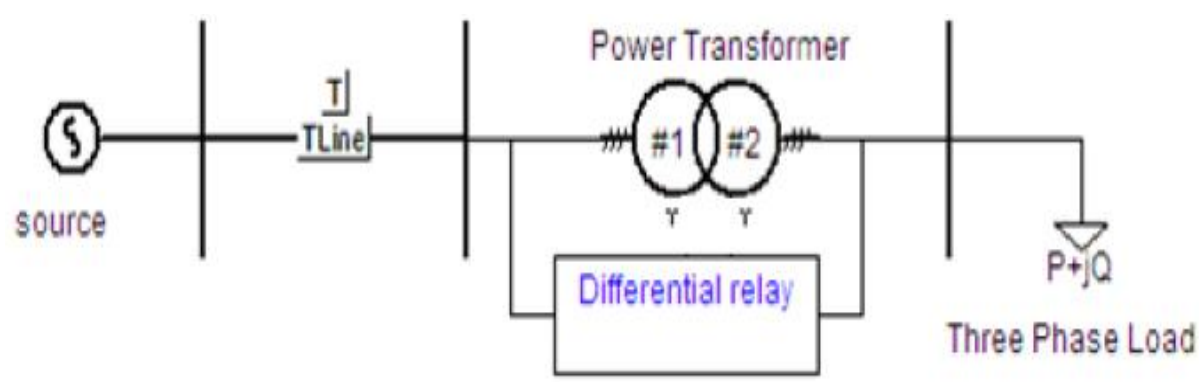

Figure 1. A single-line Diagram of the Sample Power System 


\section{The Simulation and the Laboratory's Results}

It is assumed that the converter is connected to the secondary coil without any charge. The inrush current flows only on the primary coil that may activate the variable relay. Although the magnitude of this flow is only 1 to 2 percent of the rated flow, which is subject to the condition of the stable state operation of the converter, it may increase like the numerous times that the flow is graded, when the converters are charged. In this situation, the cross-sectional angle of the voltage source is zero and the waveform of this mode is shown in Figure 2. The proposed algorithm correctly detects the converter electricity with maintain the blocked relay.

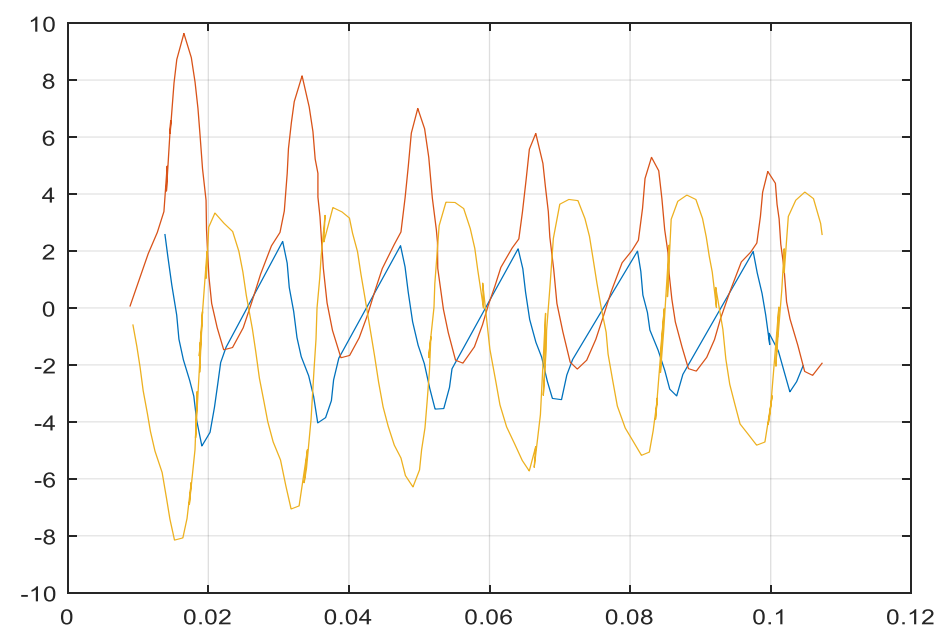

Figure 2. Displaying the Inrush Current of the Converter

\section{THE ELECTRICITY OF THE CONVERTER UNDER THE INFLUENCE OF THE ERROR}

This occurs when the power converter is related to the existence of a few prior internal errors. A practical condition that can be found hardly, especially when the error is in the coil Y, is located near the neutral part. Figure 3 provides three phase modes of the variable current for the connection conditions under the 5\% error of the secondary coil in the state A. The intersection angle of the voltage source 180 is $8.34 \mathrm{~ms}$. In this case, the algorithms detected an error state on the first data processing page $16.6 \mathrm{~ms}$. Figure 4 shows the response of the $\sigma$-gradient algorithm in the study of this problem. It is clear that the standard derivative of the variable current for all states under the threshold of angular variation indicate short-circuit occurrence.

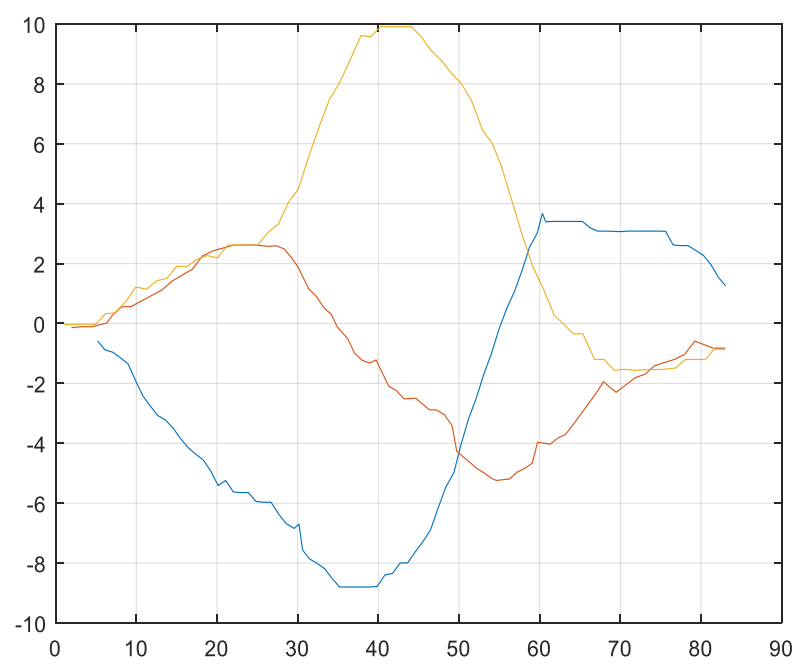

Figure 3. Displaying the Inrush Current of the Converter Electricity under the Influence of Error 


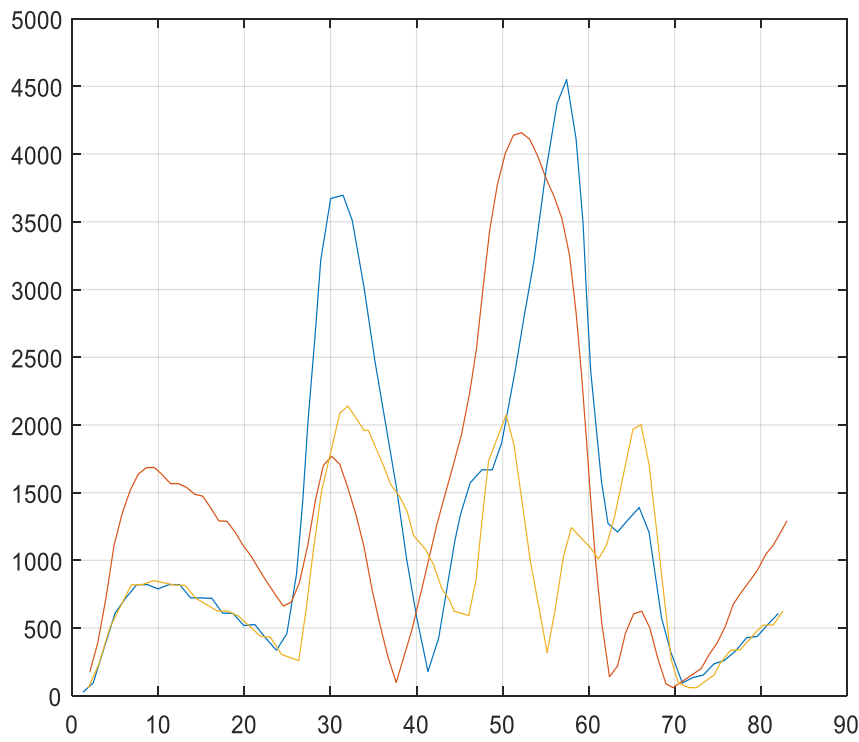

Figure 4. The Response of the $\sigma$-gradient Algorithm of the Converter Electricity under the Error Effect

\section{COMPARISON WITH THE CONVENTIONAL METHOD}

In order to validate the proposed method, the proposed methods are compared with the conventional method used in conventional relays (based on the harmonic constraints). For the conventional method, the variable current signal is processed in three modes using the Fast Fourier Transform (FFT) in order to find the harmonic elements of the signal. Based on the obtained results, it has been proven that the proposed algorithms are capable of performing the rapidly detection between the inrush current from the internal error current in an average time less or approximately equal to the half loop after the created disorder, this provides a better result. In contrast, the conventional method in the most events showed an activation time close to one loop with a time more than twice of the obtained times from the recommended methods. The output results of the algorithm is shown in Figure 5 and proposed algorithm in Figure 6. Figure 7 shows the comparison of the benchmark values for all practical examples in the field of inrush current.

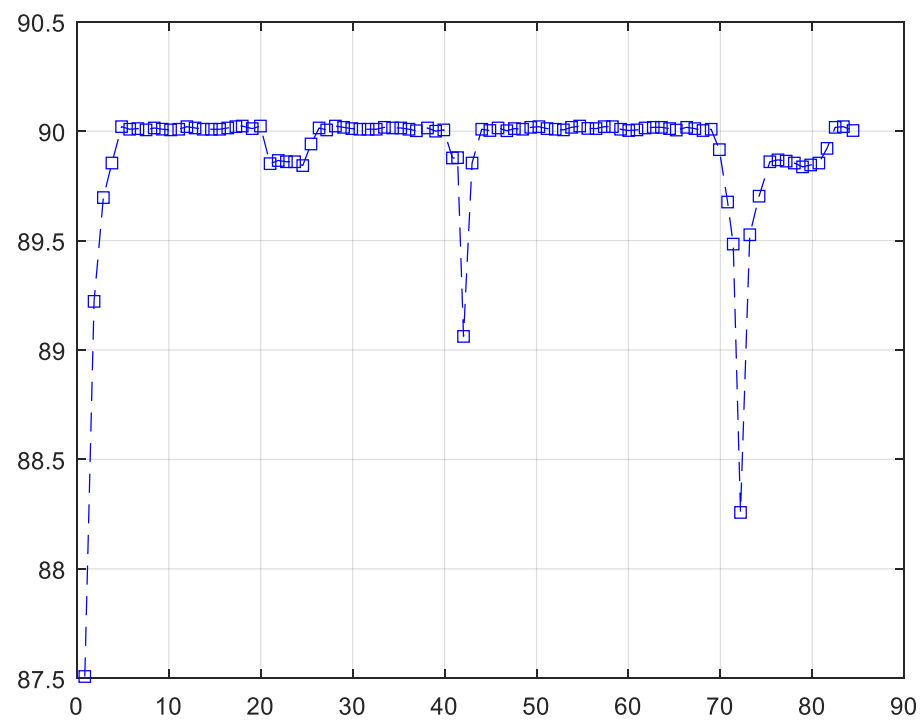

Figure 5. The Output Results of the Algorithm 


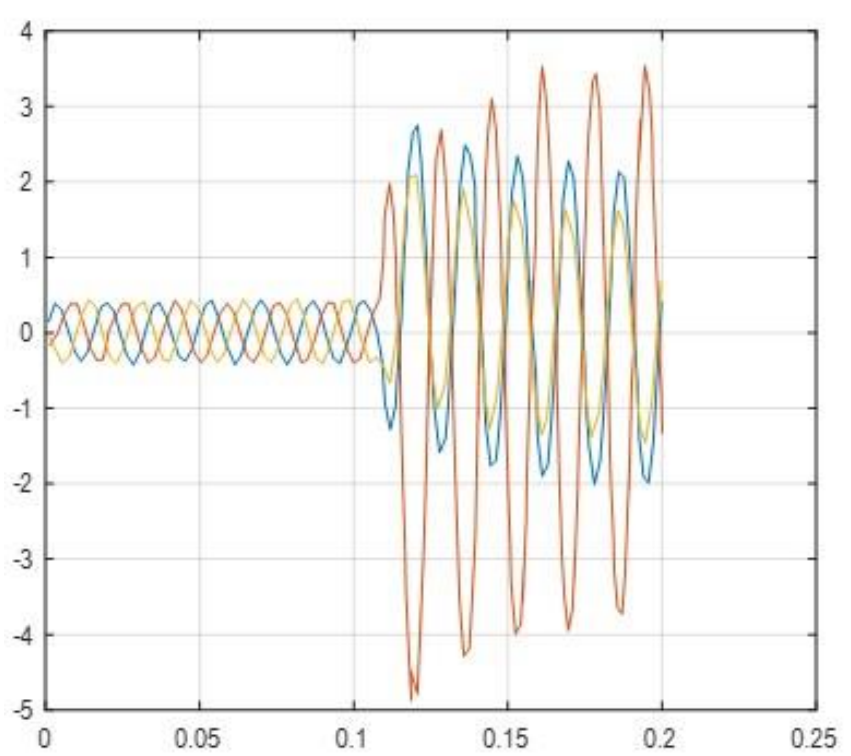

Figure 6. The Outputs Results of the Proposed Algorithm
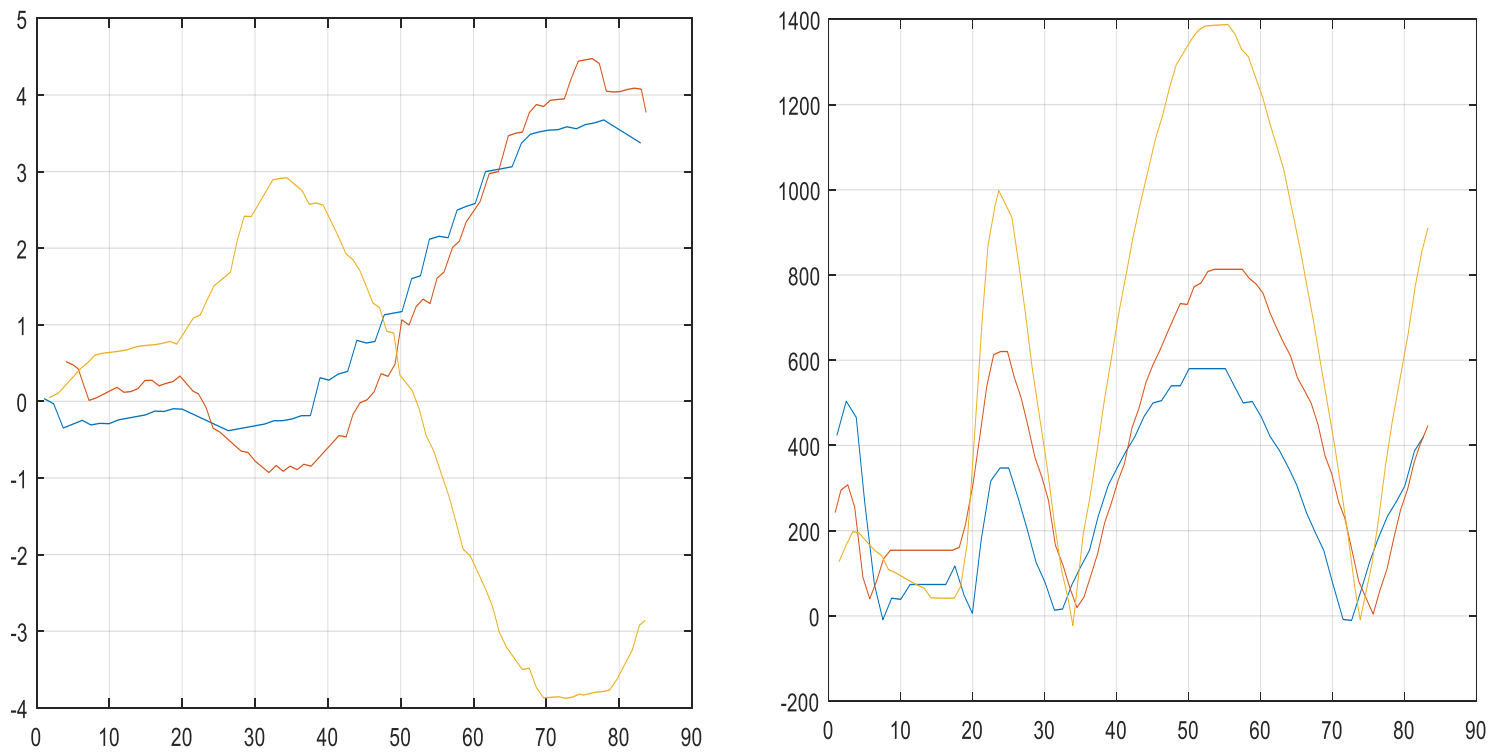

Figure 7. Comparison of the Benchmark Values for All Practical Examples in the Field of Inrush Current

\section{COMPARISON OF THE DIFFERENT METHODS}

Table 1 shows the results of applying the introduced index on different methods of detecting the inrush current from the internal error current of the transformer. We examine the results obtained by this table in the following part.

This section of Table 1 presents the results of the proposed technique. This algorithm shows satisfactory results. In addition to the prevention from the occurrence of the internal errors in the face of various situations, and the installation of the transformer in both the practical mode and the simulation, detects the condition of the transformer correctly, it has a reasonable margin (with an average of 0.8954 in the practical cases and 0.6376 in the simulated cases). Since this algorithm only needs the shape of the signal in the detection process, and independent from its scope and transformer parameters, it does not require different thresholds when facing different systems. This is one of the most important features of this algorithm. Although not the 
shortest time among the methods, the half-cycle detection time is acceptable and suitable (the speed of detection is acceptable).

Table 1. The Results of the Inrush Current

\begin{tabular}{cccccccccccc}
\hline Method & \multicolumn{2}{c}{$\begin{array}{c}\text { The minimum } \\
\text { error detection } \\
\text { time (s) }\end{array}$} & \multicolumn{2}{c}{$\begin{array}{c}\text { The average error } \\
\text { detection time (s) }\end{array}$} & \multicolumn{2}{c}{ percentage error } & \multicolumn{2}{c}{$\begin{array}{c}\text { Minimum relative } \\
\text { margin }\end{array}$} & \multicolumn{2}{c}{$\begin{array}{c}\text { Minimum relative } \\
\text { margin }\end{array}$} & threshold \\
& $\mathrm{A}$ & $\mathrm{B}$ & $\mathrm{A}$ & $\mathrm{B}$ & $\mathrm{A}$ & $\mathrm{B}$ & $\mathrm{A}$ & $\mathrm{B}$ & $\mathrm{A}$ & $\mathrm{B}$ & \\
\hline $\begin{array}{c}\text { Proposed } \\
\text { algorithm }\end{array}$ & 0.01 & 0.01 & 0.01 & 0.01 & 0 & 0 & 0.1605 & 0.6974 & 0.6376 & 0.8954 & Similar \\
$\begin{array}{c}\text { Previous } \\
\text { algorithm }\end{array}$ & 0.02 & 0.02 & 0.0155 & 0.02 & 6.17 & 22.86 & 0.0121 & 0.1316 & 0.2313 & 0.6272 & difference \\
$\begin{array}{c}\text { Previous } \\
\text { algorithm }\end{array}$ & 0.0077 & 0.0255 & 0.0053 & 0.0030 & 0 & 25.71 & 0.2667 & 0.0161 & 0.8981 & 0.1263 & difference \\
\hline
\end{tabular}

$\mathrm{A}=$ simulation

$\mathrm{B}=$ practical

\section{CONCLUSION}

As it mentioned, the basis of the detection method is based on wavelet transform and correlation coefficient. According to the results of the simulation, the energy of the wavelet transform coefficients in the inrush current and the internal error current of the transformer have different behavior, which this difference is used in the detection algorithm. The vectors obtained from the energy of the wavelet transform coefficients in the error current are not significantly different for different time cycles. and in terms of the standard a statistic is used which are correlated, but in the case of the inrush current of these energy vectors, there are many differences in each sampling window, and in other words, they are not interconnected. Regarding this property, the correlation coefficients in the error current have a stable behavior and only include a sudden decrease at the time of the error onset. While the inrush current of the correlation coefficients have oscillatory behavior and the oscillatory changes continue until the full subsidence of the inrush current. Accordingly, the criterion for detecting, if the number of deviations in the correlation coefficients exceeds the number one corresponding to the start time of the turbulence, the corresponding algorithm considers the turbulence in the current signal to be inrush current and impedes the operation of the relay; Otherwise, the turbulence is considered to be an transformer internal error and an command for turning off the transformer (which has error) would be sent.

\section{Suggestions}

a. Due to the fact that the techniques based on the wavelet transformation are sensitive to noise signals, it is suggested that the effects of these signals on the inrush current detection method are considered and the necessary measures should be taken to eliminate the effect.

b. It is proposed that model be solved using meta-innovative algorithms in order to solving the large-size samples and the samples in the real environment.

c. Using new ideas such as the use of voltage signals in this field, quantitative activities are carried out, and if a voltage-based method can be expressed without dependence on transformer parameters, we can overcome to the defects associated with the other methods.

d. The use of signal shapes can also be useful in the detection process. There are many techniques that express the similarity between the two signals. One-dimensional local binary pattern method can be one of these methods.

\section{REFERENCES}

[1] Abdollah Kamyab, Yousef Pourzadeghdoudi, Mohsen Asghari Jafarabad, Amir Taherpour Kalantari, "The Effect of Intelligent Power Transformer Injury in the Post-202,400 Power Plant Birjand," Twenty-Ninth International Electrical Conference, Nov 2014.

[2] Alireza Moradi, Mahmoud Ebadian, Mohammad Kazem Daribari, "Inrush Flow Detection from Power error Transformers Using the Gravitational Search Algorithm, Scientific Journal of the Intelligent Systems in Electrical Engineering, vol. 1, no. 1, pp. 43-58, 2010.

[3] Fatemeh Heidari, Mohammad Mirzaei, Khalil Gorgani Firouzjah, Abdolreza Sheikholeslami, "Detection of inrush current from the error current in power transformers using simultaneous flow and flux," Twenty-second annual International Power Conference 2008.

[4] IEEE, IEEE Std C37.91TM-2008 IEEE Guide for Protecting Power Transformers, 2008.

[5] M. Tripathy, R.P. Maheshwari, H.K. Verma, "Power transformer differential pro-tection based on optimal probabilistic neural network," IEEE Transactions onPower Delivery, vol. 25, no. 1, pp. 102-112, 2010. 
[6] Masoumeh Rasoolpour, Mahdi Banedeh and Alireza Ahmadifard, "Correlation Coefficient for inrush Detection from Internal Short-Circuit Current Using Wavelet Transform in Differential Protection of Power Transformer," Intelligent Systems in Electrical Engineering, vol. 4, no. 2, 2013.

[7] P. Sun, J. F. Zhang, Q. H. Wu, And S. Potts. "Morphological Identification Of Transformer Magnetizing Inrush Current." Electronics Letters, vol. 38. no. 9, pp. 437-438, Apr 2002.

[8] R.J.N. Alencar, U.H. Bezerra, "Power transformer differential protection throughgradient of the differential current," Journal of Control, Automation and Electri-cal Systems vol. 24, no. 1-2, pp. 162-173, 2013.

[9] R.W. Patterson, W.P. McCannon, G.L. Kobet, "A consideration of inrush restraintmethods in transformer differential relays," in: 54th Annual Georgia Tech Pro-tective Relaying Conference, May 2000.

[10] S. Dowdy, S. Wearden, D. Chilko, Statistics for Research, 3rd ed., John Wiley \&Sons, Inc., Hoboken, NJ, 2004.

[11] Y. Sun, J. D. Huang, And D. Z. Chen. "Detection Of Inrush Current Based On Wavelet Transform And Lvq Neural Network." In International Conference On Transmission And Distribution Conference And Exposition, IEEE Pres, Pp 1-5, New Orleans, La, Usa, Apr 19-22, 2010.

[12] Z. G. Hao, B. H. Zhang, And Y. L. Chu. Study On Transformer Protection Principle Based On Equivalent Circuit Equilibrium Equation. In Proceedings Of The Csee, 2006.

[13] Z. Lu, W. H. Tang, T. Y. Ji, And Q. H. Wu. "A Morphological Scheme For Inrush Identification In Transformer Protection." IEEE Transactions On Power Delivery, vol. 24, no. 2, pp. 560-568, Apr 2009. 